\title{
Internet of Diagnostic Things: Emerging Horizon towards Precision and Digital Health Care
}

\author{
Vijay Verma', Abhisek Mishra ${ }^{2}$, Vaishali Bisht ${ }^{2}$ and Jyoti Bala* \\ 'Aristocrat Technologies, Noida Sector 16,Uttar Pradesh, India; vijayverma090@gmail.com \\ ${ }^{2}$ Rapture Biotech International (P) Ltd., Sector 10, Noida - 201301, Uttar Pradesh, India; \\ abhishekmishra9658@gmail.com, rapturebio@gmail.com,jbjnu786@gmail.com
}

\begin{abstract}
Digital revolution and advancement of information technologies, smart phone industry, multi-omic technologies, availability with $5 \mathrm{G}$ networks and emergence of big data era has greatly impacted on diagnostic, biomedical and e-healthcare sectors. Intelligent sensors, wearable, digital imaging, smart phone based diagnostic and connected networking provides smart approach and platform towards precision diagnostics. Recently, Internet of Things (IoT) have shown remarkable impact on diverge sectors such as digital imaging, smart sensing, digital diagnostic, digital biotech, telemedicine, precision farming, lab-on phone, smart home, smart city planning, smart retails and Research and Development (R\&D) automation etc. Application of IoT technology in diagnostic and healthcare opens a way for personalization in the medical care depending on individual needs, additionally giving option for well-organized way for remote healthcare and management. This review focuses on theoretical, technological, advanced aspects of digital diagnostic utilizing IoT technology. Furthermore, we have also highlighted the opportunities and challenges for IoT in exploring the futuristic scope and innovation in diagnostic application. IoT based diagnostic, sensing and imaging devices provides unprecedented precision, automation in the diagnostic and thereby could significantly improve the biomedical and e-healthcare area.
\end{abstract}

Keywords: Digital Biomarkers, Digital Diagnostic, e-Health, Integrated Networking, Internet of Things

\section{Introduction}

The Internet of Things (IoT) is an immense concept with broad applications. IoT is a promising area with technological, industrial, social, and economic impact. Consumer products, durable goods, cars and trucks, industrial, utility components, sensors, and everyday objects are being combined with internet connectivity and potent data analytic capabilities that promise to transform the way we work and live Figure $1^{1-4}$. Projections for the economical scope of IoT are noteworthy, with some anticipating as 100 billion connected IoT devices and a global economic impact of approximate $\$ 11$ trillion predicted by 2025. The IoT usually consists of portable/non-portable devices, networks, protocols, connections, clouds, software, deep learning algorithms, analytics databases etc ${ }^{5-6}$. IoT technology brings a bridge to connect, shares the data from device, instruments and appliances to portable devices (mobile and small accessories) with internet ${ }^{7-9}$. Recent advancement in technologies has enabled us to closely monitor patient's health in real-time and remote fashion. Smart wearable such as wristbands could help to monitor vitals parameter such as heart-rate, blood sugar, blood pressure and body fat measuring weighing scales are some examples of wearable technologies ${ }^{10-12}$. Smart wristband attached

*Author for correspondence 
with health app can help in healthcare monitoring. Comprehensive international healthcare wearable market was approximately of $\$ 5$ billion in 2016 and expected to reach value of over 12 billion by 2021 (Markets and Markets).Wearable smart devices consist of infused devices and sensors which can be worn on the human body (such as: smart wristbands, watches, necklaces, headbands, belts, eyeglasses, shoes, shirts, caps, and etc. $)^{13-}$ ${ }^{15}$ these devices assist in collecting data and transferring it to software/databases and subsequently analyzed based on gathered insights and information. This can facilitate to alert the individual wearing the smart device or associated doctor to take best associated decision for the prevention, care and treatment. Additionally, IoT in healthcare is addressing and solving several biomedical challenges. IoT healthcare devices could improve the lives of millions people worldwide. IoT in healthcare provides personalized attention from medical professionals with remote options. With IoT devices supports in counting calories, sensing sugar level, heart rate, blood pressure levels, remind patients of their appointments and other medical care. In-vivo implantable devices permit a lasting monitoring option, eventually leading to efficient treatment. Researchers have shown leadless pacemaker for such application ${ }^{16}$. This is well established that IoT have applications for clinical patient monitoring and treatment. A system-level approach for both human factor and the technical aspects of networking, databases and privacy are managed to achieve such chosen relevance. Progression in the fields like micro-electronics and in the micro-technologies significantly impacted modernization in e-medicine. Advancement in such technologies has provided an expansion and the development of miniaturization of size of the devices applied for sensing, imaging and diagnosis ${ }^{17-18}$. Moreover, its connection with IoT technologies resulted in proficient, better data processing of "big-data" associated with the diagnosis and health monitoring purpose. IoT diagnostic brings innovation and intelligent system and platform which not only benefits patients but doctors as well. IoT based digital diagnostic makes remote diagnosis possible even if the patient is at home or if the doctors are at different hospital or clinic which is going to break geographical barriers. Advancement of technology and its application associated with IoT based sensors, portable devices, wearable devices, and smart phones device/apps facilitate evolution in diagnostic sector. In past decades, several studies were reported showing IoT based implication towards smart and precision diagnostics of infectious and chronic diseases ${ }^{19-21}$. Here we have highlighted the concept, advancement and application of "Internet of Diagnostics Things" (IoTD). Molecular biomarkers based advanced diagnostic are complex and rapidly growing areas in the era of multi-omic technologies, sequencing revolution and big data time ${ }^{22-24}$. Digital diagnostic is an advanced and pioneering technology that has improved biomedical and health sector. In current era where technologies are changing at faster rate and interdisciplinary co-dependence of medical sector on diverse areas like; biotechnology, chemist, physics, mathematics, information technology, data scientist, nanotechnology etc is also facing the impact of this revolution. Advancement in theses sector has also impact the way conventional diagnostic, medical care and healthcare were previously seen, hence to deliver better services, adaptation and improvement of health sector with fast emerging technologies are must.

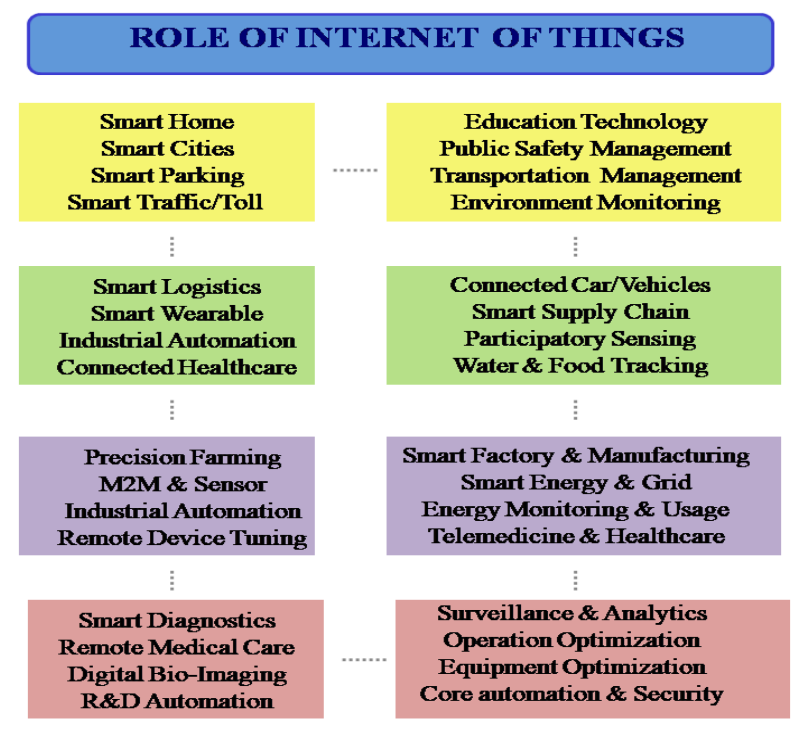

Figure 1. Overview of various applications of Internet of Thing (IoT) with potential scope in diverge sectors. IoT has wide spread applications in many fields.

\section{Conceptual Perspective of Internet of Things (IOT)}

IoT system usually consists of sensors/devices connected via cloud or high speed connectivity. Significant raw data get transferred to the cloud, various advanced/updated software are used for data analysis and later assist in 
decision making and taking related chosen downstream decision ${ }^{25-26}$. Furthermore, this could support in sending an alert or automatically adjusting the sensors/devices, if it's required, thereby resulting in automation and providing tool for efficient monitoring system. The crucial aspects that IoT system and platform provides are better connectivity, analyzing options, integrating, automation and active engagement. Technically, IoT system/platforms could connect computing devices, embedded devices, wearable, mechanical and digital machines, objects, animals or people, given with unique identifiers and the ability to transfer data over a network excluding the requirement of human-to-human or human-to-computer interaction ${ }^{3,27-28}$. Fundamentally, in current time as smartphone has made revolutionary transformation and given diverge application scope with the use of internet, likewise theoretically any device or things could be connected via internet and used for various automated and innovated relevance. Theses connected devices/systems to the internet can send information or receive information, creating a way for easier, smarter and innovative applications. This provides a tool for real-time, automatic, safe, efficient way for decisions making based on collected data. Briefly, the IoT can be classified into significant components or building blocks such as app devices, app for IoT verticals/app generator, connectivity enablement (i.e. SIM/e-SIM management), analytics (data storage, analysis and reporting). Such system and platform requires management in terms of configuration, control, provisioning, security, authentication, monitoring and software updates ${ }^{4,29,30}$. Basically IoT system consists mainly of 4 parts as illustrated in (Figure 2). The main four components are: a. Devices: usually has one or more sensor to detect the particular parameter and subsequently could send the information to cloud network (platform), b. Internet connectivity: devices are connected to the internet and online platform. Wifi enabled device, wifi enabled modem, broadband connection. Ethernet and internet are popularly used. The connection between the sensor/devices are through cloud and variety of method such as cellular, satellite, wifi, LPWN (Low Powered Windarea Network) are connected with internet or Ethernet, c. IoT Platform: These platform provide way for data collection, software, algorithms, analysis tool, systems to process and make decisions based on this data. Briefly, this comprise of repository databases with crucial data, external interfaces (APIs, SDKs and gateways that provides interface for third party system (e.g. ERP, CRM). Algorithms for calculation and analytics, machine learning are also used ${ }^{31-32}$. Additional tools, apps, access management, reporting systems are some optional part of such platforms which help in device management, data visualization, processing and management, and $\mathbf{d}$. Applications: Integration with applications such as online services, web apps, mobile apps etc. The application is the interface between the user and the platform to monitor, configure or control the device and also could interact with the application.

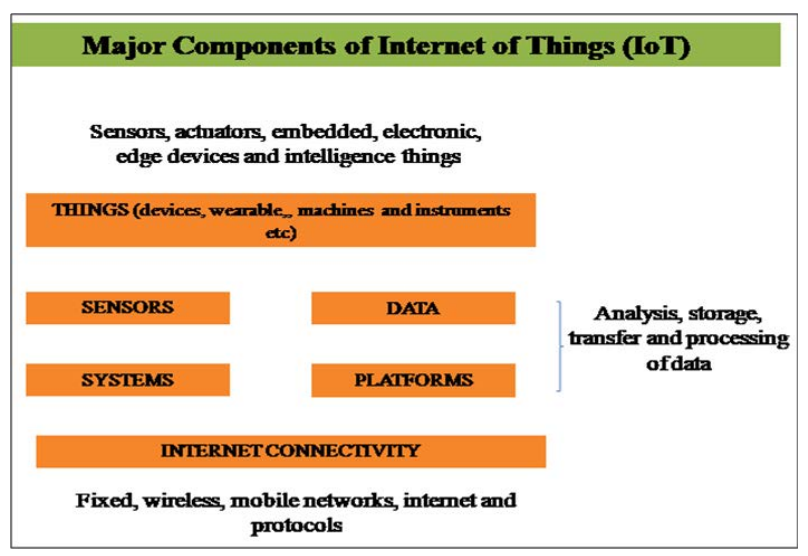

Figure 2. Representation of major components of Internet of Thing (IoT) and functionality.

\section{Role of Internet of Things (IOT)}

IoT have recently shown promising progression in various sectors such as: home automation (smart home), smart cities, manufacturing sector, energy, environment, precision farming/agriculture automation, logistic, automation of $\mathrm{R} \& \mathrm{D}$, and e-health (including digital pathology, imaging, diagnostic and digital health) ${ }^{33-37}$. Additionally, connected car, connected health, smart supply chain and retails have also been improved with the expansion of IoT $T^{1,3,38,39}$. Smart farming based IoT technologies are an advanced method to grow food in cleaner and sustainable way. This method applies contemporary information and communication technologies to agriculture, targeted not only to reduce waste but by enhancing agricultural productivity to an optimum level. Using the IoT technology for the identification of diseases in plants is also beneficial in monitoring a large field of crops. IoT has diverse applications in medicine from remote observing to intelligent sensors and smart medical devices ${ }^{40-42}$. These can prevent and monitor patients, as well as improve the quality of medical care. Sensor, wearable, wireless network, 
actuators, or devices in diagnostic lab, instrumentation, imaging equipment, digital camera and networked through computer systems generates huge data in diagnostic and bio-imaging sector. Digital diagnostic and imaging has gained lot of consideration for healthcare improvement. Various Io $\mathrm{T}$ based devices and platforms have been explored in various imaging, sensing and diagnostic purpose. Digital diagnostic and health are associated with IoT technology, that offer advantages over conventional methods with respect to cost effectiveness, precision, consistency, efficiency, real time monitoring and lesser requirement with human effort in healthcare. IoT results in improving data analysis, quality of imaging, accurate sensing, real-time monitoring, automation, sharing the data and subsequently executing chosen functions. Due to increasing population and fast changing modern lifestyle there is also enhancement in the diseases and there are several fields of medical area that's needs innovation and development to cope with these challenges. Diseases such as chronic diseases, diabetes cancers, AIDS tuberculosis, heart attacks etc require novel, proficient, portable system and device for prevention and diagnosis for these diseases. In health sector smart and timely tracking of clinical product and donor organ for transplant can be smartly tracked in transit via IoT devices. If some deviation/ error or damage been noticed could be easily notify to the user end providing real time monitoring and digital tracking options. In pharmaceutical sector IoT have been anticipated and offer product quality testing at various levels of manufacturing and for product optimization. There are several reports that show the application of IoT based wireless devices such as blood glucose sensors, real time glucose monitor, smart insulin pens, insulin pumps, and closed-loop systems etc for diabetes ${ }^{43-46}$. IoT based nasal airflow sensor used to monitor the airflow rate of a patient who is in a need of respiratory support. This consists of a flexible thread that fix behind the ears and two prongs are placed in the nostril, through which breathing of the patient is measured. Constant health monitoring can offer information about the status of physiological indicators (such as, blood pressure, pulse, blood sugar, calories, and etc.). Moreover, the development of invasive IoT based diagnoses devices could be user friendly, easy to use and precise with diagnosis. IoT-enabled portable and smart devices in dropping support costs and could bring potential point of care diagnostic.

\section{Internet of Diagnostic Things}

Diagnostic, sensor and imaging devices and systems make use of intelligent smart devices to connected IoT network for sensing, testing, diagnosis, and monitoring and analysis purpose and thereby help in prediction of diseases and its complexities, subsequently managing and caring the patients. IoT components typically consist of devices, wearable, embedded electronics, software, sensors, actuators, and network connectivity that enable these objects to collect, store, share and exchange data. The key benefits of IoT for diagnostic sector are low cost, reducing visits to doctor and hospital, remote monitoring, improved outcome of treatment, reliable and accurate diagnostic data, automation and overall better disease management (Figure 3). Telemedicine, healthcare management, clinical operations, diagnostic labs, and bio-imaging imaging are some segments of health market while Bluetooth, Wi-Fi, NFC, Zigbee, RFID are few networks which could be used for IoT based digital health. Moreover IoT provides proactive mean to patient and healthcare professional with real time data availability and better analysis for effective treatment. IoT brings efficient, remote diagnosis option for automated health assessment that can be assessed virtually or electronically. Several researchers and companies have developed smartphone based devices and software for analytics platform development used for diagnostic, sensing and imaging leading to remote diagnostic possible. Enabling remote diagnostic and powerful analytics, Flex era Software and Glass beam can be used for retrieval and analysis of $\log$ files to enable companies to do remote diagnostics on IoT enabled devices. Applications enabled by IoT are significant in diagnostic sector which facilitate an evolution in the diagnostic sector and subsequently in e-medicine.

Yang, et al. (2018) have proposed an IoT based remote pain monitoring system by utilizing facial surface Electro-Myo-Gram (sEMG) and cloud platform. In this study, a wearable wireless device with bio-sensing ability was used to observe pain intensity of a patient by utilizing facial sEMG. Wireless sensor node (upto up to eight channels of sEMG), integrated into an IoT system for pain monitoring. This system could be use concurrent pain data collection, analysis and transfer to a mobile web application. This system provides effective 


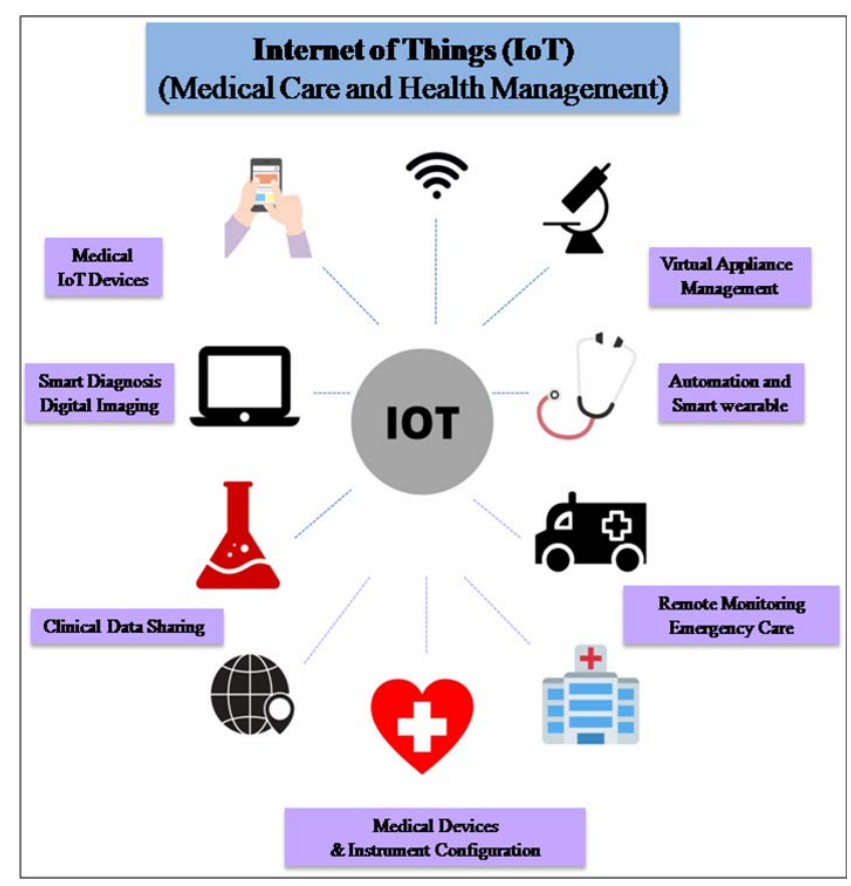

Figure 3. Internet of Thing (IoT) and it's diverge application in healthcare application.

way of real-time streaming of high-volume sEMG data, digital signal processing, interpreting, and visualization using sensor node, web browser, wireless communication and web application on cloud platform. This study concludes a scalable IoT system for real-time monitoring and a wearable solution which has potential scope as an automatic pain assessment via facial expressions ${ }^{47}$. Millana, et al. (2018) has demonstrated an integrated IoT intelligent system for the automatic detection of cardiac variability. Detection of abnormal cardiac events during clinical examination is infrequent. They have proposed the implementation and assessment of a mobile based system for real-time monitoring and detection of cardiovascular problems related to heart-rate variability. Briefly, they have integrated an IoT e-Health kit based on Arduino and validated the algorithms for heart rate variability to build cost valuable, reliable and scalable solution. Additionally, a mobile system based on an Android application which integrates the Pan and Tompkins algorithm and a module to retrieve real-time electrocardiography through Bluetooth interface was utilized ${ }^{48}$. Several studies have been reported that implements IoT to carry out digital diagnostic. Lifelens Project is one such example. Researchers have developed a mobile phone application that can be used for malaria diagnosis. This technological system provides point-of-care tool that could have enormous impact on malaria diagnosis and treatment. This is a robust mobile diagnostic solution for malaria that have shown 94.4 per cent accuracy rate when applied to imagining blood samples provided by different labs. Briefly, Lifelens has replaced conventional way of malaria testing which were earlier done by unreliable cotton swab with a new approach with highdefinition, high-magnification camera (magnification upto 350 times), supported with better image analysis software to advance the precision diagnosis ${ }^{49}$. A drop of blood as sample drawn from person and placed on slide, later digitally examined through the lens with the use of attachment for higher magnification and resolution. The smear is imaged, and then diagnosed using the Lifelens application that can be easily downloaded to the phone. This tool gives opportunity to patient with the minimum amount of training so they can user friendly diagnose. Recent promising platform like IoT provide advantage by providing connection of any physical object, for example a smart phone devices and medical instruments, to the internet resulting in better applications in the field of digital pathology, digital biomarker, digital imagining, digital diagnostic and e-healthcare.

Interestingly, Kostikis, et al. (2015) have proposed a practical smart-phone-based tool to accurately assess upper limb tremor in PD patients. In this study they have used 25 PD patients and 20 age related healthy controls using smart-phone accelerometer and gyroscope. Authors suggest, this method could provide effective tool in assisting doctors for monitoring and additionally remote evaluation is also possible by such system ${ }^{50}$. Pasluosta,et al. (2015) has also reported significance of IoT in the management of PD using smart wearable technologies ${ }^{51}$. Additionally, Klimova and Kuca (2018) have reported role of IoT in the assessment, diagnostics and treatment of Parkinson's Disease (PD). Chronic neurological disorders such as PD need medical attention. Development of efficient and affordable strategies are required to address challenge related $\mathrm{PD}$ diagnosis and monitoring. Their findings suggest that IoT has impending scope in healthcare platform by providing, an efficient, cost-effective and affordable approach in the management of PD and other chronic neurological disorders ${ }^{52}$. Gait impairments related with disabling symptoms during musculoskeletal and neurological conditions are common. Wearable/devices for gait sensors are crucial with diagnostic potential for gait and recently been emerging as promising tool for monitoring, 
tutoring and guiding gait execution. Ferrari, et al. (2016) have reported novel test method for estimating spatiotemporal gait parameters using smart-phone, shoe-worn inertial wearable sensors tool for accurate diagnosis ${ }^{53}$. Precisely, they have reported implementation of a zerovelocity-update gait analysis system based on Kalman filter and off-the-shelf shoe-worn inertial sensors. The algorithms were used for analysis of gait events and step length estimation was accordingly designed to comply with pathological gait patterns with specificity. Additionally, android app was introduced to sustain fully wearable and stand-alone real-time gait analysis. Another study was performed by Ellis, et al. (2015) to test and show the potential of a smart-based mobile app, Smart MOVE used in the assessment of gait and gait variability in PD. These studies consist of smart phone, heel-mounted footswitch sensors, sensor-embedded walkway for testing and assessment. To summarize their findings, their data suggest a smart-based app which could serve as a suitable assessment method gait analysis ${ }^{54}$.

Istepanian, et al. (2011) has shown IoT potential impact for non-invasive glucose level sensing. Diabetes is a major chronic disease and of global concern. Interestingly, first time IoT based diabetes management by non-invasive glucose level sensing with advanced opto-physiological assessment technique was reported in this study. This study proposes way to accomplish an intelligent identification and management in a heterogeneous connectivity environment from the mobile healthcare outlook. Additionally, this also enable smart communication connectivity routes between mobile patients and care services through innovative IP based networking architectures ${ }^{55}$. Klonoff (2017) has reported appealing role of Fog computing and Edge computing architectures for processing data from connected devices has valuable impact on diabetes management. The advantages of using Fog computing and Edge computing compared to cloud computing is they provide better speed with data transmission, lower cost associated, lesser dependency on limited bandwidths and greater privacy and security. These connected diabetes devices with Fog computing or Edge computing provides effective and fast way tool for diabetes patients medical care ${ }^{56}$. Diabetes and long-neglected Diabetic Foot Ulcer (DFU) and its related complications are common and of major global concern. Basatneh, et al. (2018) has reported health sensors and IoT potential for diabetes care and management. Briefly they have shown the advancement and boom of IoT along with smart devices, smart phone based monitoring/sensing system. The med-tech industry is moving toward homecare revolution, which has potential for developing effective solutions with noteworthy prospective to trim down DFUassociated costs and providing better patients care ${ }^{57}$.

Various diseases require surgery for treatment. Image-Guided Surgery (IGS) is a surgical process where surgeons use an invasive tracked instrument to make a preoperative image. IGS helps the surgeons to obtain better results during the surgical process by getting images from different area at different times and angles, subsequently resulting in better planning, guidance, positioning of the surgical device, and any postoperative processes. The components of IGS are workstation, navigation tool, patient's images dataset and all these are connected through the internet. The success of the surgery is based on the direct visualization of the target area and the tracking tool. Before surgical treatment, different images of the target area are taken by suitable imaging modalities such as magnetic resonance imaging, computed tomography, ultrasound and positron emission tomography. The distinct images of the same area acquired in different periods need to be aligned into one image, as the information of a single image is not sufficient for the situation of the target area inside the body and for decision making. The process of aligning images into a single image is known as image registration. Second crucial component of the IGS is the tracking tool, a tool that shows the real-time position and situation of the medical device throughout the surgical process. IGS tracking tools compromise of optical, mechanical position pointer, electromagnetic tracking tool and connected with IoT helps in critical surgical conditions ${ }^{58}$. Additionally, Precision diagnosis for the monitoring (realtime/ remote) of disease prognosis at initial stage could provide effective prevention, cure, effective treatment and thereby overall healthcare management. Several companies and startups (such as EarlySense and Monica Healthcare) focus on connected biometric sensors for clinical purpose. Interestingly, Quanttus, MC10, and others devices are under development with clinical-grade properties and expecting for FDA approval. Furthermore, clinical IoT equipment, Eyenetra, a smart phone-enabled "auto-refractor" for vision testing has also been reported. Interestingly, IoT opens innovative horizon in health management and healthcare by providing efficiency via development of clinically relevant diagnostic approach. IoT devices/systems can be developed as clinically grade biometric sensor/portable wearable. 


\section{Discussion}

Interestingly in $2020,40 \%$ of IoT related technologies are health-related as IoT provide advantages in terms of ease with connectivity, effective device management, and better data analytics with health data, deep learning algorithm and accuracy eventually with associated decision making and medical care management. IoT system and platforms help to smartly use, transfer, store and analyze the huge and immense data generated in various sectors. With systematic and logical use of cloud computing, sensor and actuator, these data can be applied remotely or real times purpose by distributed servers. Effective, portable, easy to handle sensors with cost-effective solutions to measure our vital are in demand ${ }^{59-60}$. The current challenges are making IoT practices adaptable, friendly and implementable in biological sector like diagnostic, imaging, $\mathrm{R} \& \mathrm{D}$ and healthcare by reducing the gap between biologist and IoT technologies. IoT has opened digital transformation of home-based patient medical care for various diseases, as it enables patient engagement, personalized care and smart management through efficient, telecommunication, data mining, and inclusive feedback tailored to patient requirements (Figure 4). Moreover, with recent advances in voice-activated commands technology, smart phone based devices and its integration with of IoT have given innovative opportunities to improve the patient's central role and responsibility in managing and enabling toward digital health care ecosystem. Sudha, et al. (2018) have summarized medical diagnosis based on IoT using Arduino which has enormous implication on healthcare sector. This makes faster communication and better analysis. Now days, doctor can access the patient's vital information, pulse rate and temperature values through the web page, connected devices and the data could be shared with patient and for real-time/remote analysis based on patient need ${ }^{60}$. There are studies that show the sensor application based on IoT that allows patient/user to measure the body temperature. These temperature sensors have immense medical significance and patient health care. Besides, there are reports where other vitals sensing such as blood pressure have been shown. These device can monitor and record fluctuation in blood pressure, additionally can also check the systolic pressure (as the heart beats) over the diastolic pressure (as the heart relaxes between the beats) ${ }^{61}$. With miniaturization of sensing/ diagnostic devices, furthermore with advancement in information technology, multi-omics, sequencing revolution, data science, and faster telecommunications, impacts innovation in R\&D and biomedical sector leading to a revolution towards precision, point-of-care and digital diagnostic. IoTM/IOTD could support better medical care by enabling patients to spend less time with their medical practitioner, though IoT also has few challenges and drawbacks. The number of medical devices and the enormous amounts of data they gather can be a problem for a medical center's IT group to oversee, manage, store, transfer, related security and analysis of such huge data. Additionally there are pressure and issue of how to keep the patient information secure, particularly when it is being transferred between different devices. IoT for remote medical care and health monitoring is now established area and growing tremendous with great medical scope. With advancement of technologies in various fields, devices based on implantable electronics, IoT and integration of bio-interfaces, devices and sensors have recently reported for several applications for healthcare. Implantable biomedical devices, senor, diagnostic system, radiofrequency devices, circuits, bio-imaging system and systems provide efficient and intelligent path for data communications, diagnosis, imaging, monitoring and analysis. Several such devices, circuits and smartphone based app systems for non-invasive diagnosis were also reported ${ }^{62}$. Additionally, sensing devices, imaging instruments, digital camera, diagnostic chips, intelligent connected IoT based devices and their application in several cancer screening and infectious disease detection could be used for advanced diagnostic.

\section{Challenges and Future Scope}

It is well establishes that IoT plays great role in industrial revolution and impacting several sector and benefiting our lives tremendously. This area holds potential scope for innovation and automation and thus exploring novel application based on IoT hold promise in R\&D, diagnostic and healthcare sector too. Though there are few challenges that need to be address such as scalability issue due large volumes of data processing, technological complexity, design based challenge, government regulatory involvement, data safety, privacy and related ethics. There are several reports that show that uses block chain technology for security issues and challenges in IoT. Additionally, evolutionary computation and other computational intelligence were also reported for artificial 
immune mechanism for IoT security architecture, data mining/fusion in IoT enabled cyber physical systems, and data driven cybersecurity. Cloud and IoT based disease prediction and diagnosis system could bring revolution in diagnostic sector, minimizing the cost and leading the diagnostic towards precision, personalized, point-of care and digital health era. IoT technology could bring real-time production performance analysis and precision diagnosis model for several disease models. In the era of personalized and precision medicine, big data are being generated from multi omics, imaging, sensing and R\&D labs. Hence dependency of biomedical, clinical and healthcare sector on computational scientists, data scientist are greatly required in current era to reduce the gap and develop superior tools to control, integrate and share data to make it medically significant. Outstandingly, IoT promise a notable impact on medical diagnostic and healthcare providing a bridge to reduce the gap among biologist, medical personal, patients and technologist leading towards the digital healthcare era, improving patient's lives globally.

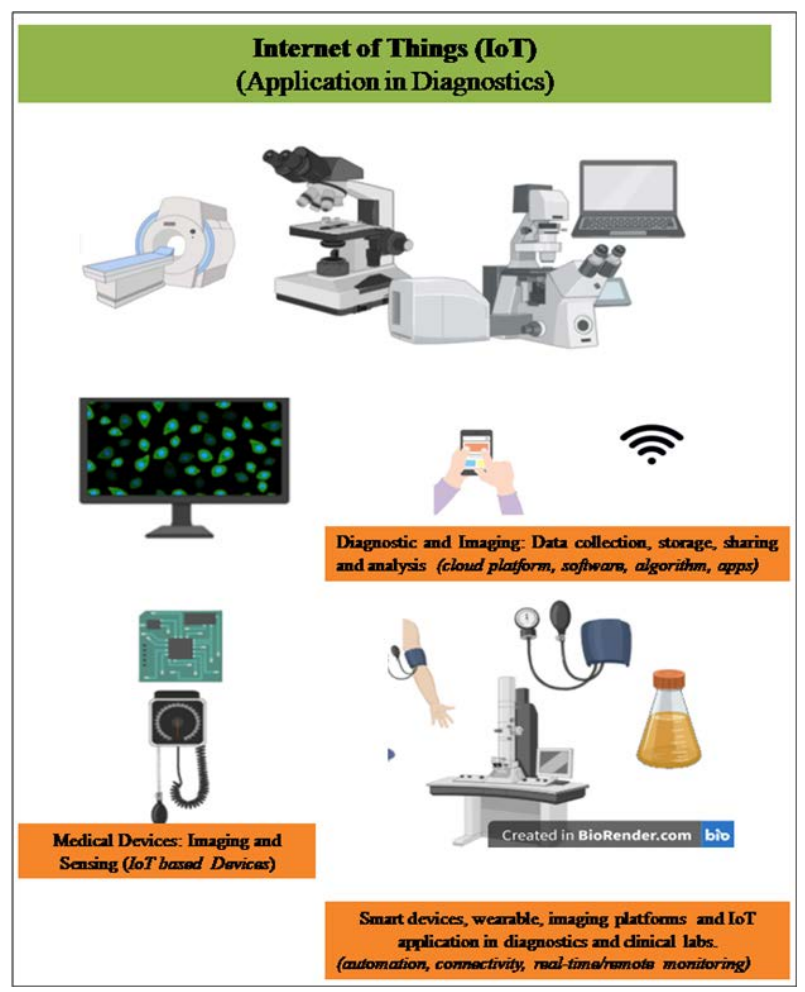

Figure 4. Implication and prospects of Internet of Thing (IoT) and its application in diagnostic sector with the advancement of microelectronic, sensor technology, digital imaging, optics, information technology, IoT and multi- omics and their utilizations at diagnostic centre subsequently results in automation, innovation and precision in diagnostic and healthcare sector resulting in real-time and remote healthcare management.

\section{Conflict of Interest}

The authors declare no conflict of interest.

\section{References}

1. $\mathrm{Xu} \mathrm{DL}, \mathrm{He} \mathrm{W}, \mathrm{Li}$ S. Internet of things in industries: $\mathrm{A}$ survey, IEEE Transactions on Industrial Informatics. 2014; 10(4):2233-43. https://doi.org/10.1109/TII.2014.2300753.

2. Basatneh R, Najafi B, Armstrong DG. Health sensors, smart home devices, and the internet of medical things: An opportunity for dramatic improvement in care for the lower extremity complications of diabetes, J. Diabetes Sci. Technol. 2018; 12(3):577-86. https://doi. org/10.1177/1932296818768618. PMid: 29635931, PMCid: PMC6154231.

3. Vongsingthong S, Smanchat S. Internet of things: A review of applications and technologies, Suranaree J. Sci. Technol. 2014; 21(4):359-74.

4. Hassan QF. Internet of Things A to Z: Technologies and Applications. John Wiley \& Sons; 2018. p. 27-28. https:// doi.org/10.1002/9781119456735.

5. Gubbi J, Buyya R, Marusic S, Palaniswami M. Internet of Things (IoT): A vision, architectural elements, and future directions, Fut. Gen. Comp. Syst. 2013; 29(7):1645-60. https://doi.org/10.1016/j.future.2013.01.010.

6. Mahmood Z. Connected Environments for the Internet of Things: Challenges and Solutions, Springer; 2018. p. 89-90. https://doi.org/10.1007/978-3-319-70102-8.

7. Sadek RA. An agile Internet of Things (IoT) based Software Defined Network (SDN) architecture, Egypt Comp. Sci. J. 2018; 42(9):13-29.

8. Silva $\mathrm{BN}$, Khan $\mathrm{M}$, Han $\mathrm{K}$. Internet of things: $\mathrm{A}$ comprehensive review of enabling technologies, architecture, and challenges, IETE Tech. Review. 2018; 35(2):205-20. https://doi.org/10.1080/02564602.2016.1276 416.

9. Zorzi M, Gluhak A, Lange S, Bassi A. From today's intranet of things to a future internet of things: A wirelessand mobility-related view, IEEE Wireless Comm. 2017; 17(6):44-51. https://doi.org/10.1109/MWC.2010.5675777.

10. Nayyar A, Puri V. Data glove: Internet of Things (IoT) based smart wearable gadget, J. of Advances in Math. and Comp. Sci. 2016; 15(5):1-12. https://doi.org/10.9734/ BJMCS/2016/24854. 
11. Thierer AD. The internet of things and wearable technology: Addressing privacy and security concerns without derailing innovation, Richmond J. of Law and Techn. 2015; 21(2):6. https://doi.org/10.2139/ssrn.2494382.

12. Hiremath S, Yang G, Mankodiya K. Wearable Internet of Things: Concept, architectural components and promises for person-centered healthcare. 2014 th Int. conf. on Wireless mob. Comm.. and Healthcare. (MOBIHEALTH), Athens, Greece. doi: 10.1109/ MOBIHEALTH.2014.7015971. https://doi.org/10.4108/icst.mobihealth.2014.257440.

13. Putri AO, Ali MA, Saad M, Hidayat SS. Wearable sensor and internet of things technology for better medical science: A review, Int. J. of Eng. and Techn. 2018; 7(4):11. https://doi. org/10.14419/ijet.v7i4.11.20677.

14. Fernández-Caramés TM, Fraga-Lamas P. Towards the Internet of smart clothing: A review on IoT wearables and garments for creating intelligent connected e-textiles, Electronics. 2018; 7(12):405. https://doi. org/10.3390/electronics7120405.

15. Castillejo P, Martínez JF, López L, Rubio G. An internet of things approach for managing smart services provided by wearable devices, Int. J. of Dis. 2013; 9(2). https://doi. org/10.1155/2013/190813.

16. Amar AB, Kouki AB, Cao H. Power approaches for implantable medical devices, Sensors. 2015; 15(11):28889914. https://doi.org/10.3390/s151128889. PMid: 26580626, PMCid: PMC4701313

17. Nasri F, Moussa N, Mtibaa A. Smart mobile system for health parametersfollowshipbasedon WSNandandroid, WorldCong. on Comp. and IT. 2013. doi: 10.1109/WCCIT.2013.6618733. https://doi.org/10.1109/WCCIT.2013.6618733.

18. Biswas S, Gogoi AK. Design issues of piezoresistive MEMS accelerometer for an application specific medical diagnostic system. 2nd IEEE Int Conf on Emerg Elect (ICEE), IETE Techn. Review. 2016; 33(1):11-16. https://doi.org/10.1080/ 02564602.2015.1065713.

19. Ullah K, Shah MA, Zhang S. Effective ways to use Internet of Things in the field of medical and smart health care, Int. Conf. on Intel. Syst. Engin. 2016. doi: 10.1109/INTELSE.2016.7475151. https://doi.org/10.1109/INTELSE.2016.7475151.

20. Robinson DC, Mohanty S, Young J, Jones G, Wesemann D. Novel techniques for mapping infectious diseases using point of care diagnostic, Sens. Phy. and Tech. of Sens. (ISPTS), 2015 2nd Int Symp., Pune; 2015. p. 325-27. https:// doi.org/10.1109/ISPTS.2015.7220139. PMid: 25524761.

21. Sareen S, Sood SK, Gupta SK. IoT-based cloud framework to control Ebola virus outbreak, J. of Amb. Intell. and Hum. Comp. 2018; 9(3):459-76. https://doi.org/10.1007/s12652016-0427-7. PMid: 32218876, PMCid: PMC7091278.

22. Akter S, Wilshire G, Davis JW, Bromfield JJ, Crowder S, Joshi T, Pelch KE, Schust DJ, Meng A, Barrier B, Nagel SC. A multi-omics informatics approach for identifying molecular mechanisms and biomarkers in clinical patients with endometriosis. 2017 IEEE Int. Conf. on Bioinfo. and Biomed; 2017. https://doi.org/10.1109/ BIBM.2017.8218003.

23. Zhan X, Zhou T, Cheng T, Lu M. Recognition of multiomicsbased molecule-pattern biomarker for precise prediction, diagnosis, and prognostic assessment in cancer, Bioinfo. Tools for Det. and Clin. Interp. of Gen. Var. 2019; 1-22. https://doi.org/10.5772/intechopen.84221.

24. Burmester GR. Rheumatology 4.0: Big data, wearables and diagnosis by computer, Annals of the Rheum. Dis, 2018; 77:963-65. https://doi.org/10.1136/ annrheumdis-2017-212888. PMid: 29802224, PMCid: PMC6029631.

25. Lueth KL. IoT basics: Getting started with the Internet of things, White paper. 2015; 1-9.

26. Truong HL, Dustdar S. Principles for engineering IoT cloud systems, IEEE Cloud Comp. 2015; 68-76. https://doi. org/10.1109/MCC.2015.23.

27. Desai P, Sheth A, Anantharam P. Semantic gateway as service architecture for IoT interoperability, 2015 IEEE Int. Conf. on Mob. Serv.; 2015. p. 313-19. https://doi. org/10.1109/MobServ.2015.51.

28. Fortino G, Guerrieri A, Russo W, Savaglio C. Integration of agent-based and cloud computing for the smart objectsoriented IoT, 2014 IEEE 18th Int. Conf. on Comp. Supported Coope Work in Desig; 2014. https://doi.org/10.1109/ CSCWD.2014.6846894.

29. Sundaravadivel P, Kougianos K, Mohanty SP, Ganpathiraju MK. Everything you wanted to know about smart health care: Evaluating the different technologies and components of the Internet of Things for better health, IEEE Cons. Elect. Mag. 2018; 7(1):18-28. https://doi.org/10.1109/ MCE.2017.2755378.

30. Chun S, Seo S, Oh B, Lee KH. Semantic description, discovery and integration for the Internet of things. Proceedings of the 2015 IEEE 9th Int. Conf. on Semantic Comp; 2015. 27275. https://doi.org/10.1109/ICOSC.2015.7050819. PMid: 25381844.

31. Broring A, Schmid S, Schindhelm CK, Khelil A, Kabisch S, Kramer D, Phuoc DL, Mitic J, Anicic D Teniente E. Enabling IoT ecosystems through platform interoperability, IEEE Software, 2017. https://doi.org/10.1109/MS.2017.2.

32. Keramidas G, Voros N, Hübner M. Components and Services for IoT Platforms, Springer; 2016. Doi: 10.1007/978-3-31942304-3. https://doi.org/10.1007/978-3-319-42304-3.

33. Xu G, Shi Y, Sun X, Shen W. Internet of things in marine environment monitoring: A review, Sensors, 2019; 19:1711. https://doi.org/10.3390/s19071711. PMid: 30974791, PMCid: PMC6479338.

34. Guo S, Qiang M, Luan X, Xu P, He G, Yin X, Xi L, Jin X, Shao J, Chen X, Fang D, Li B. The application of the Internet of 
things to animal ecology, Integr. Zool. 2015; 10(6):572-78. https://doi.org/10.1111/1749-4877.12162. PMid: 26338071.

35. Gope P, Hwang T. BSN-care: A secure IoT-based modern healthcare system using body sensor network, IEEE Sens. J. 2016; 16(5):1368-76. https://doi.org/10.1109/ JSEN.2015.2502401.

36. Rateni G, Dario P, Cavallo F. Smartphone-based food diagnostic technologies: A review, Sensors (Basel). 2017; 17(6). https://doi.org/10.3390/s17061453. PMid: 28632188, PMCid: PMC5492046.

37. Swaroop KN, Chandu K, Gorrepotu R, Deb S. A health monitoring system for vital signs using IoT, Internet of Things. Elsevier. 2019; 5:116-129. Doi 10.1016/j. iot.2019.01.004. https://doi.org/10.1016/j.iot.2019.01.004.

38. Evans D. The Internet of things: How the next evolution of the internet is changing everything, CISCO White Paper. http://www.cisco.com/web/about/ac79/docs/innov/IoT_ IBSG_0411FINAL.pdf.

39. Chen S., Xu H., Liu D., Hu B., Wang H. A vision of IoT: Applications, challenges, and opportunities with china perspective, IEEE Int. of Things J. 2014; 1(4):349-59. https:// doi.org/10.1109/JIOT.2014.2337336.

40. Doukas C, Maglogiannis I. Bringing IoT and cloud computing towards pervasive healthcare, 2012 6th Int. Conf. on Inn. Mob. and Internet Serv. in Ubiq. Comp. https://doi.org/10.1109/IMIS.2012.26.

41. Smys S, Chen JZ. Special issue on medical imaging technologies for IoT based wireless patient monitoring, Cur. Med. Imag. 2019; 15(8):711. https://doi.org/10.2174/ 157340561508190927122912 . PMid: 32008539.

42. Shah R, Chircu A. IoT and AI in healthcare: A systematic literature review, Issues in Inf. Syst. 2018; 19(3):33-41.

43. Sangave NA, Aungst TD, Patel DK. Smart connected insulin pens, caps, and attachments: A review of the future of diabetes technology, Diabetes Spect. 2019; 32(4):378-84. https://doi.org/10.2337/ds18-0069. PMid: 31798296.

44. Istepanian RSH, Jovanov E, ZhangYT. Guest editorial introduction to the special section on m-health: Beyond seamless mobility and global wireless health-care connectivity, IEEE Trans. Inf. Technol. Biomed. 2004; 8(4):405-14. https://doi.org/10.1109/TITB.2004.840019. PMid: 15615031.

45. Yanchapaxi, C. Tipantuña, Calderón X. Wearable System for Monitoring of Human Physical Activities, Proc. of the 4th Int. Conf. on eDem. and eGov.; 2017. p. 245-50. https://doi.org/10.1109/ICEDEG.2017.7962543.

46. Chen LB, Chang WJ, Su JP, Ciou JY, Ciou YJ, Kuo CC, Li KS. A wearable-glasses-based drowsiness-fatigue-detection system for improving road safety. 2016 IEEE 5th Global Conference on Consumer Electronics; 2016. https://doi. org/10.1109/GCCE.2016.7800456.
47. Yang G, Jiang M, Ouyang W, Ji G, Xie H, Rahmani AM, Liljeberg $\mathrm{P}$, Tenhunen $\mathrm{H}$. IoT-based remote pain monitoring system: from device to cloud platform, IEEE J. Biomed. Health Inform. 2018; 22(6):1711-19. https://doi. org/10.1109/JBHI.2017.2776351. PMid: 29990259.

48. Millana AM, Palao C, Llatas CF, de Carvalho P, Bianchi AM, Traver V. Integrated IoT Intelligent System for the Automatic Detection of Cardiac Variability. Conf. Proc. IEEE Eng. Med. Biol. Soc.; 2018. p. 5798-801.

49. Grimberg BT, Grimberg KO. Hemozoin detection may provide an inexpensive, sensitive, 1-minute malaria test that could revolutionize malaria screening, Expert Rev. Anti. Infect. Ther. 2016; 14(10):879-83. https://doi.org/10 .1080/14787210.2016.1222900. PMid: 27530228, PMCid: PMC5224914.

50. Kostikis N, Hristu-Vasakelis D, Arnaoutoglon M, Kotsavasiloglou C. A smartphone-based tool for assessing Parkinson hand tremor, IEEE J. Biomed. Health Inform. 2015; 19(6):1835-42. https://doi.org/10.1109/ JBHI.2015.2471093. PMid: 26302523.

51. Pasluosta CF, Gassner H, Winkler J, Klucken J, Eskofier BM. An emerging era in the management of Parkinson's disease: Wearable technologies and the Internet of Things, IEEE J, Biomed, Health Inform. 2015; 2168-94. https://doi. org/10.1109/JBHI.2015.2461555. PMid: 26241979.

52. Klimova B, Kuca K Internet of things in the assessment, diagnostics and treatment of Parkinson's disease. Health and Technology. 2018; 9:87-91. https://doi.org/10.1007/ s12553-018-0257-z.

53. Ferrari A, Ginis P, Hardegger M, Casamassima F, Rocchi L, Chiari L. A mobile Kalman-filter based solution for the real-time estimation of spatial-temporal gait parameters, IEEE Trans. Neural. Syst. Rehabil. Eng. 2016; 24(7):76473. https://doi.org/10.1109/TNSRE.2015.2457511. PMid: 26259246.

54. Ellis RJ, Ng YS, Zhu S, Tan DN, Anderson B, Schlaug G, et al. A validated smartphone-based assessment of gait and gait variability in Parkinson's disease, PLoS One. 2015; 10(10):e0141694. https://doi.org/10.1371/journal. pone.0141694. PMid: 26517720, PMCid: PMC4627774.

55. Istepanian $\mathrm{R}, \mathrm{Hu} \mathrm{S}$, Philip N, Sungoor A. The Potential of Internet of M-Health Things "m-IoT" for Non-Invasive Glucose Level Sensing, Conf. Proc. IEEE Eng. Med. Biol. Soc.; 2011. p. 5264-66. https://doi.org/10.1109/ IEMBS.2011.6091302. PMid: 22255525.

56. Klonoff DC. Fog computing and edge computing architectures for processing data from diabetes devices connected to the medical internet of things, J. Diabetes Sci. Technol. 2017; 11(4):647-52. https://doi. org/10.1177/1932296817717007. PMid: 28745086, PMCid: PMC5588847. 
58. Vijayalakshmi S. Image-guided surgery through internet of things, Internet of Things in Biomed Eng. 2019; 75-116. https://doi.org/10.1016/B978-0-12-817356-5.00005-X.

59. Lee KA, Yim E, Kim A. Medical diagnosis based on IOT using Arduino, Ind. J. of Pub. Health R\&D. 2018, 9(12):270859. https://doi.org/10.5958/0976-5506.2018.02157.5.

60. Sudha S, Shruthi P, Sharanya M. IoT based measurement of body temperature, Int. Res. J. of Eng. and Tech. 2018; 05(3):3913-15. https://www.irjet.net/archives/V5/i3/ IRJET-V5I3916.pdf.
61. Dias D, Silva Cunha. JP. Wearable health devices-vital sign monitoring, systems and technologies, Sensors. 2018; 18:2414-. https://doi.org/10.3390/s18082414. PMid: 30044415, PMCid: PMC6111409.

62. Heinemann L, Krämer U, Klötzer HM, Hein M, Volz D, Hermann M, Heise T, Rave K. Noninvasive Glucose measurement by monitoring of scattering coefficient during oral glucose tolerance tests, Diabetes Tech. \& Ther. 2000; 2(2):211-20. https://doi.org/10.1089/15209150050025168. PMid: 11469261. 